\title{
A stable range description of the space of link maps
}

\author{
Thomas G GoodwiLlie \\ BRIAN A MUNSON
}

\begin{abstract}
We study the space $\operatorname{Link}(P, Q ; N)$ of link maps: maps from $P \sqcup Q$ to $N$ such that the images of $P$ and $Q$ are disjoint. We identify the homotopy fiber of the inclusion $\operatorname{Link}(P, Q ; N) \rightarrow \operatorname{Map}(P, N) \times \operatorname{Map}(Q, N)$ in a stable range, showing that it has a $(2(n-p-q)-3)-$ connected map to the infinite loopspace of a certain Thom spectrum.
\end{abstract}

57Q45; 58D15, 57R99

\section{Introduction}

Let $N$ be a smooth manifold and let $P$ and $Q$ be smooth compact manifolds. A (smooth) link map of $P$ and $Q$ in $N$ is a pair $(f: P \rightarrow N, g: Q \rightarrow N)$ of smooth maps such that $f(P)$ is disjoint from $g(Q)$. The set of link maps, denoted by $\operatorname{Link}(P, Q ; N)$, is an open subspace of $\operatorname{Map}(P, N) \times \operatorname{Map}(Q, N)=\operatorname{Map}(P \sqcup Q, N)$.

For brevity we will write $\mathcal{M}$ for $\operatorname{Map}(P, N) \times \operatorname{Map}(Q, N)$ and denote the complement of the set of link maps in $\mathcal{M}$ by $\mathcal{B}$. We prove that a certain "linking number" map

$$
\ell: \operatorname{hofiber}_{\left(f_{1}, g_{1}\right)}(\mathcal{M}-\mathcal{B} \rightarrow \mathcal{M}) \rightarrow \Omega Q_{+}^{T N-(T P \oplus T Q)} \operatorname{holim}\left(P \stackrel{f_{1}}{\rightarrow} N \stackrel{g_{1}}{\leftarrow} Q\right)
$$

is $(2(n-p-q)-3)$-connected, where $p, q$ and $n$ are the dimensions of the manifolds. The map was defined by the second author in [5], although the version we reference below is of a more homotopy-theoretic flavor, and is given by Klein and Williams [3]. Its domain is the homotopy fiber of the inclusion $\mathcal{M}-\mathcal{B} \rightarrow \mathcal{M}$ with respect to any point $\left(f_{1}, g_{1}\right) \in \mathcal{M}-\mathcal{B}$. Its codomain is the infinite loopspace associated to the Thom spectrum of a virtual vector bundle. Both of these spaces are $(n-p-q-2)-$ connected. In the case when $p+q=n-1$ it was shown in [5] that the effect of the map $\ell$ on $\pi_{0}$ can be interpreted as a generalized linking number.

Functor calculus (the manifold version developed by Weiss [6] and the first author and Weiss [2]) offers one point of view on link maps. Consider the functor $(U, V) \mapsto$ $\operatorname{Link}(U, V ; N)$ whose domain is the poset $\mathcal{O}(P \sqcup Q)=\mathcal{O}(P) \times \mathcal{O}(Q)$ of open subsets of $P \sqcup Q$. Its best linear approximation is $\operatorname{Map}(U, N) \times \operatorname{Map}(V, N)$. Our result can 
be interpreted as a statement about a quadratic approximation to the same functor, but we will not pursue this here. This work overlaps the recent work of Klein and Williams; in particular, some of the material in Section 3 also appears in [3].

Our main result is:

\section{Theorem 1.1 The map}

$$
\Lambda: \Sigma \text { hofiber }(\mathcal{M}-\mathcal{B} \rightarrow \mathcal{M}) \rightarrow Q_{+}^{T N-(T P \oplus T Q)} \operatorname{holim}(P \rightarrow N \leftarrow Q)
$$

adjoint to $\ell$ is $(2(n-p-q)-1)-$ connected.

The fact that $\ell$ is $(2(n-p-q)-3)$-connected then follows immediately by the Freudenthal Theorem, since the domain of $\ell$ is $(n-p-q-2)$-connected. Note that the connectivity claimed for $\Lambda$ is negative if $p+q \geq n$, so it is no loss to assume $p+q<n$.

\subsection{Conventions}

A space $X$ is $k$-connected if for every $j$ with $-1 \leq j \leq k$ every map $S^{j} \rightarrow X$ can be extended to a map $D^{j+1} \rightarrow X$. In other words, $(-1)$-connected means nonempty and if $k \geq 0$ then $k$-connected means that there is exactly one path-component and that the homotopy groups vanish through dimension $k$. A map is $k$-connected if each of its homotopy fibers is $(k-1)$-connected. A (weak) equivalence is an $\infty$-connected map.

We write $Q X=\Omega^{\infty} \Sigma^{\infty} X$ if $X$ is a based space. If $X$ is unbased, then $X_{+}$means $X$ with a disjoint basepoint added and $Q_{+} X$ means $Q\left(X_{+}\right)$. For a vector bundle $\xi$ over a space $\mathrm{X}$, the unit disk bundle and the unit sphere bundle are $D(X ; \xi)$ and $S(X ; \xi)$. The Thom space $X^{\xi}$ is the quotient $D(X ; \xi) / S(X ; \xi)$, or equivalently the homotopy cofiber of the projection $S(X ; \xi) \rightarrow X$. If $\xi$ and $\eta$ are two vector bundles on $X$, then by choosing a vector bundle monomorphism $\eta \rightarrow \epsilon^{i}$ to a trivial bundle we can define $Q_{+}^{\xi-\eta} X=\Omega^{i} Q X^{\xi \oplus \epsilon^{i} / \eta}$. This is essentially independent of the choice of $i \geq 0$ and vector bundle monomorphism, in the sense that for large $i$ the weak homotopy type of this space is independent of those choices.

\section{Sketch of the proof of Theorem 1.1}

To prove Theorem 1.1 we will use the diagram (1) below and obtain the connectivity of $\Lambda$ from the connectivities of all the other maps. For this we must introduce another closed set $\mathcal{V} \subset \mathcal{B}$. Recall that a point $(f, g) \in \mathcal{M}$ belongs to $\mathcal{B}$ if the statement $f(x)=z=g(y)$ holds for some pair $(x, y) \in P \times Q$ and some point $z \in N$. The 
closed set $\mathcal{B}$ has codimension $n-p-q$ in $\mathcal{M}$ in some sense. Inside this space $\mathcal{B}$ of "bad" maps is a set $\mathcal{V}$ of "very bad" maps, having codimension $2(n-p-q)$ in $\mathcal{M}$. A point $(f, g)$ is in $\mathcal{V}$ if either the statement $f(x)=z=g(y)$ holds for more than one choice of $(x, y, z)$ or else it holds for one such choice in such a way that the associated map of tangent spaces $T_{x} P \oplus T_{y} Q \rightarrow T_{z} N$ is not injective. The set $\mathcal{B}-\mathcal{V}$ may be regarded as a submanifold of $\mathcal{M}$. It has maps to $P, Q$ and $N$ given by $x, y$ and $z$. Pulling back tangent bundles via these maps, we obtain vector bundles on $\mathcal{B}-\mathcal{V}$, which we will denote simply by $T P, T Q$ and $T N$. There is also a monomorphism $T P \oplus T Q \rightarrow T N$, and its cokernel $T N /(T P \oplus T Q)$ may be thought of as the normal bundle of $\mathcal{B}-\mathcal{V}$ in $\mathcal{M}$.

The next result immediately implies Theorem 1.1.

Theorem 2.1 In the homotopy commutative diagram below, the maps $F$ and $H$ are equivalences, the maps $G, C$ and $D$ are $(2(n-p-q)-1)$-connected, and the map $E$ is $(3(n-p-q)-2)-$ connected.
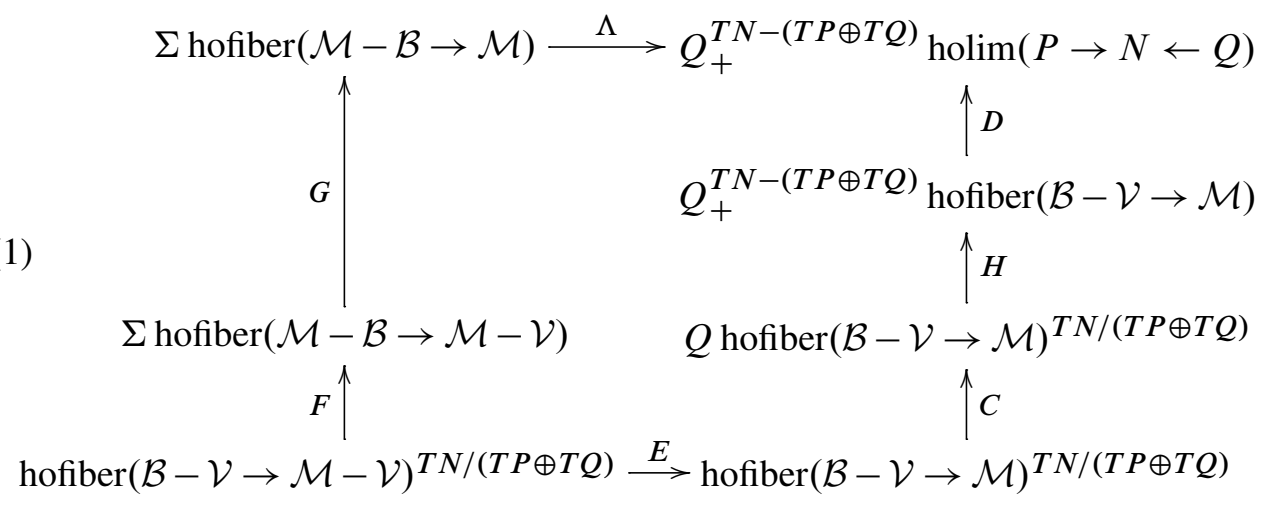

We now briefly define the maps in the diagram and explain about their connectivities. Steps that are sketchy here will be filled in the following sections. Let $c=n-p-q$. The equivalence $F$ is essentially an instance of the following general fact. If $Y$ is a smooth submanifold of $X$ and also a closed subset, then the suspension of the homotopy fiber of the inclusion $X-Y \rightarrow X$ is equivalent to the Thom space, over the homotopy fiber of $Y \rightarrow X$, of the normal bundle of $Y$ in $X$. This general fact will be proved, and adapted to the present function-space setting, in Section 4.

The map $G$ is an inclusion map. Since $\mathcal{V}$ has codimension $2 c$ in $\mathcal{M}$, the inclusion $\mathcal{M}-\mathcal{V} \rightarrow \mathcal{M}$ is $(2 c-1)$-connected. (This will be worked out in detail in Section 3.) Therefore the map of homotopy fibers is $(2 c-2)$-connected and the map $G$ of suspensions is $(2 c-1)$-connected. 
The map $E$ is a map of Thom spaces. For a $k$-connected map $Z \rightarrow W$ of spaces and a vector bundle $\xi$ on $W$ with fiber dimension $d$, the associated map $Z^{\xi} \rightarrow W^{\xi}$ is $(k+d)-$ connected. In our case $d=c$ and $k=2 c-2$; the inclusion of hofiber $(\mathcal{B}-\mathcal{V} \rightarrow \mathcal{M}-\mathcal{V})$ into hofiber $(\mathcal{B}-\mathcal{V} \rightarrow \mathcal{M})$ is $(2 c-2)$-connected, again because the inclusion of $\mathcal{M}-\mathcal{V}$ into $\mathcal{M}$ is $(2 c-1)$-connected.

The map $C$ is the canonical map $Z \rightarrow Q Z$, where the space $Z$ is $(c-1)$-connected, being the Thom space of a vector bundle of rank $c$. By the Freudenthal Theorem, the map is $(2 c-1)$-connected.

The equivalence $H$ is simply a matter of rewriting the Thom spectrum of a virtual vector bundle $\xi-\eta$ as the suspension spectrum of the Thom space of $\xi / \eta$ when $\eta$ is a subbundle of $\xi$.

The map $D$ arises from a $(c-1)$-connected map from hofiber $(\mathcal{B}-\mathcal{V} \rightarrow \mathcal{M})$ to $\operatorname{holim}(P \rightarrow N \leftarrow Q)$. To explain further, we need the space $\widetilde{\mathcal{B}}$ of all $((f, g), x, y, z) \in$ $\mathcal{M} \times P \times Q \times N$ such that $f(x)=z=g(y)$. Projection to $\mathcal{M}$ gives a map from $\tilde{\mathcal{B}}$ onto $\mathcal{B}$. Let $\tilde{\mathcal{V}} \subset \widetilde{\mathcal{B}}$ be the preimage of $\mathcal{V}$. The projection $\widetilde{\mathcal{B}}-\tilde{\mathcal{V}} \rightarrow \mathcal{B}-\mathcal{V}$ is an isomorphism. The inclusion $\widetilde{\mathcal{B}}-\widetilde{\mathcal{V}} \rightarrow \widetilde{\mathcal{B}}$ is $(c-1)$-connected for reasons of codimension (again, the details are in Section 3), and therefore the induced map $\operatorname{hofiber}(\mathcal{B}-\mathcal{V} \rightarrow \mathcal{M}) \rightarrow \operatorname{hofiber}(\widetilde{\mathcal{B}} \rightarrow \mathcal{M}) \simeq \operatorname{holim}(P \rightarrow N \leftarrow Q)$ is also $(c-1)-$ connected. There are vector bundles $T P, T Q$ and $T N$ on $\widetilde{\mathcal{B}}$ pulling back to their namesakes on $\mathcal{B}-\mathcal{V}$. (The monomorphism $d f \oplus d g: T P \oplus T Q \rightarrow T N$ is not available on the $\operatorname{holim}(P \rightarrow N \leftarrow Q)$ side, which is why we switched from Thom spaces to Thom spectra).

We end this section with a brief account of the commutativity of diagram (1). First we need to define the map $\Lambda$. As mentioned in Section $1, \Lambda$ is adjoint to a map $\ell: \operatorname{hofiber}_{\left(f_{1}, g_{1}\right)}(\mathcal{M}-\mathcal{B} \rightarrow \mathcal{M}) \rightarrow \Omega Q_{+}^{T N-(T P \oplus T Q)} \operatorname{holim}(P \rightarrow N \leftarrow Q)$, which is a composite described below (also see Klein and Williams [3, Section 9]). Let $\left(f_{t}, g_{t}\right) \in$ $\operatorname{hofiber}_{\left(f_{1}, g_{1}\right)}(\mathcal{M}-\mathcal{B} \rightarrow \mathcal{M})$. The map $\mathcal{M} \rightarrow \operatorname{Map}(P \times Q, N \times N)$ given by $(f, g) \mapsto$ $f \times g$ induces a map

$$
\begin{aligned}
\operatorname{hofiber}_{\left(f_{1}, g_{1}\right)}(\mathcal{M}-\mathcal{B} \rightarrow \mathcal{M}) \rightarrow \operatorname{hofiber}_{f_{1} \times g_{1}}(\operatorname{Map}(P \times Q & \left., N \times N-\Delta_{N}\right) \\
& \rightarrow \operatorname{Map}(P \times Q, N \times N)) .
\end{aligned}
$$

We can identify the latter homotopy fiber as a space of sections as follows.

Let

$$
E=\operatorname{holim}\left(P \times Q \stackrel{f_{1} \times g_{1}}{\longrightarrow} N \times N \longleftarrow N \times N-\Delta_{N}\right) .
$$

The projection map $E \rightarrow P \times Q$ is a fibration with fiber over $(p, q)$ the space $\Phi_{2}(N)=$ $\operatorname{hofiber}_{\left(f_{1}(p), g_{1}(q)\right)}\left(N \times N-\Delta_{N} \rightarrow N \times N\right)$. Let $\Gamma(P \times Q, E)$ be its space of sections. This space of sections has a preferred basepoint given by $\left(f_{1}, g_{1}\right)$. It is equivalent 
to hofiber $f_{1} \times g_{1}\left(\operatorname{Map}\left(P \times Q, N \times N-\Delta_{N}\right) \rightarrow \operatorname{Map}(P \times Q, N \times N)\right)$ by inspection. Let $Q_{f} S_{f} E \rightarrow P \times Q$ be the fibration whose fibers are $Q S \Phi_{2}(N)$, where $S$ stands for the unreduced suspension. The canonical map $\Gamma(P \times Q, E) \rightarrow \Omega \Gamma\left(P \times Q, Q_{f} S_{f} E\right)$ is easily shown to be $(2 n-p-q-1)-$ connected, and there is an equivalence

$$
\Omega \Gamma\left(P \times Q, Q_{f} S_{f} E\right) \simeq \Omega Q_{+}^{T N-(T P \oplus T Q)} \operatorname{holim}\left(P \times Q \stackrel{f_{1} \times g_{1}}{\longrightarrow} N \times N \leftarrow \Delta_{N}\right)
$$

which is the identity on the loop coordinate. Moreover, there is a homeomorphism

$$
\operatorname{holim}\left(P \times Q \stackrel{f_{1} \times g_{1}}{\longrightarrow} N \times N \leftarrow \Delta_{N}\right) \cong \operatorname{holim}\left(P \stackrel{f_{1}}{\longrightarrow} N \stackrel{g_{1}}{\longleftarrow} Q\right) .
$$

The composite map

$$
\operatorname{hofiber}_{\left(f_{1}, g_{1}\right)}(\mathcal{M}-\mathcal{B} \rightarrow \mathcal{M}) \rightarrow \Omega Q_{+}^{T N-(T P \oplus T Q)} \operatorname{holim}\left(P \stackrel{f_{1}}{\longrightarrow} N \stackrel{g_{1}}{\longleftarrow} Q\right)
$$

is the map $\ell$, and $\Lambda$ is its adjoint.

Now let $\left(f_{t}, g_{t}, v\right) \in \operatorname{hofiber}(\mathcal{B}-\mathcal{V} \rightarrow \mathcal{M}-\mathcal{V})^{T N / T P \oplus T Q}$. Here $v$ is a vector of length $0 \leq|v| \leq 1$, and $\left(f_{t}, g_{t}, v\right)$ is identified to a point when $|v|=1$. After applying the maps $E, C, H$ and $D$ in diagram (1), it is clear that $\left(f_{t}, g_{t}, v\right)$ is sent to $\left(\left(x_{0}, \beta, y_{0}\right), v\right) \in Q_{+}^{T N-(T P \oplus T Q)} \operatorname{holim}(P \rightarrow N \leftarrow Q)$, where $\left(x_{0}, y_{0}\right) \in P \times Q$ is the unique pair such that $f_{0}\left(x_{0}\right)=g_{0}\left(y_{0}\right)$ and $\beta: I \rightarrow N$ is the path defined by $\beta(s)=f_{1-2 s}\left(x_{0}\right)$ for $0 \leq s \leq 1 / 2$ and $\beta(s)=g_{2 s-1}\left(y_{0}\right)$ for $1 / 2 \leq s \leq 1$.

Now we must apply $F, G$ and $\Lambda$ to $\left(f_{t}, g_{t}, v\right)$. A careful examination of the material in Section 4 reveals that $F$ sends $\left(f_{t}, g_{t}, v\right)$ to the point $s \wedge\left(\tilde{f}_{t}, \tilde{g}_{t}\right)$, where $s=1-|v|$ and $\left(\tilde{f}_{t}, \tilde{g}_{t}\right) \in \operatorname{hofiber}(\mathcal{M}-\mathcal{B} \rightarrow \mathcal{M})$ is defined as follows. For $s \leq t \leq 1$, we have $\left(\widetilde{f}_{t}, \widetilde{g}_{t}\right)=\left(f_{(t-s) /(1-s)}, g_{(t-s) /(1-s)}\right)$. For $0 \leq t \leq s,\left(\tilde{f}_{t}, \tilde{g}_{t}\right)$ has the following properties: $\left(\tilde{f}_{t}, \widetilde{g}_{t}\right) \in \mathcal{M}-\mathcal{B}$ for $t<s,\left(\widetilde{f}_{s}, \widetilde{g}_{s}\right)=\left(f_{0}, g_{0}\right)$ has a unique pair $\left(x_{0}, y_{0}\right) \in$ $P \times Q$ such that $f_{0}\left(x_{0}\right)=g_{0}\left(y_{0}\right)=z_{0} \in N$ and such that $f_{0}^{\prime}\left(x_{0}\right)-g_{0}^{\prime}\left(y_{0}\right) \in T_{z_{0}} N$, when projected to $T_{z_{0}} / T_{x_{0}} P \oplus T_{y_{0}} Q$, is equal to $v$ (here $f_{0}^{\prime}$ and $g_{0}^{\prime}$ are the derivatives with respect to $t$ ). From this description of $F$ and the description of $\Lambda$ above, the diagram commutes.

\section{Codimension and connectivity}

The proof outlined above uses that the pair $(\mathcal{M}, \mathcal{M}-\mathcal{V})$ is $(2 n-2 p-2 q-1)-$ connected and that the pair $(\tilde{\mathcal{B}}, \tilde{\mathcal{B}}-\tilde{\mathcal{V}})$ is $(n-p-q-1)-$ connected. We now justify these statements more carefully.

For the first, it suffices if for every smooth manifold $W$ of dimension $k<2 n-2 p-2 q$, and for every map of pairs $\phi:(W, \partial W) \rightarrow(\mathcal{M}, \mathcal{M}-\mathcal{V})$, there is a homotopy of pairs to a map that is disjoint from $\mathcal{V}$. 
Consider the adjoint map $\Phi: W \times(P \sqcup Q) \rightarrow N$. By a preliminary homotopy we can assume that $\Phi$ is smooth, and we can make the homotopy small enough in the $C^{0}$ sense so that it corresponds to a homotopy of pairs. If we can show that the condition $\phi^{-1}(\mathcal{V})=\varnothing$ holds for a dense set of all such smooth maps $\Phi$, then another small homotopy will complete the job. For the density statement we will use the multijet transversality theorem of Mather [4, Proposition 3.3] (which appears in [1] as Theorem 4.13).

Recall the setup: Two smooth maps $\Phi, \Psi: X \rightarrow Y$ have the same $m$-jet at $x \in X$ if $\Phi(x)=\Psi(x)$ and $\Phi$ and $\Psi$ have the same derivatives through order $m$. Let $X^{(r)} \subset X^{r}$ be the space of configurations of $r$ distinct points in $X$. The maps $\Phi$ and $\Psi$ have the same $m$-multijet at $\left(x_{1}, \ldots, x_{r}\right) \in X^{(r)}$ if for every $i \in\{1, \ldots r\}$ they have the same $m$-jet at $x_{i}$. The manifold $J_{m}^{(r)}(X, Y)$ of multijets has a point for each $r$-tuple $\left(x_{1}, \ldots, x_{r}\right)$ and each equivalence class of maps as above. A smooth map $\Phi: X \rightarrow Y$ determines a smooth map $j_{m}^{(r)}(\Phi): X^{(r)} \rightarrow J_{m}^{(r)}(X, Y)$. The multijet transversality theorem asserts that, for every submanifold $Z$ of $J_{m}^{(r)}(X, Y)$, the set of all $\Phi$ such that $j_{m}^{(r)}(\Phi)$ is transverse to $Z$ is a countable intersection of dense open sets in the function space $\operatorname{Map}(X, Y)$. It follows that such a set, or even the intersection of countably many such sets, is dense.

We now introduce various submanifolds $Z$ of $J_{m}^{(r)}(W \times(P \sqcup Q), N)$, for various values of $r$ and $m$. The point is that the condition $\phi^{-1}(\mathcal{V})=\varnothing$ will hold if and only if for each of these the set $j_{m}^{(r)}(\Phi)$ is disjoint from $Z$. The codimension of $Z$ will always be big enough so that in order for $j_{m}^{(r)}(\Phi)$ to be transverse to $Z$ it must be disjoint. Therefore the theorem will guarantee that there are maps $W \rightarrow \mathcal{M}-\mathcal{V}$ arbitrarily close to a given map $\Phi: W \rightarrow \mathcal{M}$.

Let $k$ be the dimension of $W$. We consider the various ways in which $\phi$ could hit $\mathcal{V}$.

(1) There might exist distinct $x_{1}$ and $x_{2}$ in $P$ and distinct $y_{1}$ and $y_{2}$ in $Q$ such that for some $w \in W$ we have $\Phi\left(w, x_{1}\right)=\Phi\left(w, y_{1}\right)$ and $\Phi\left(w, x_{2}\right)=\Phi\left(w, y_{2}\right)$. Then the point

$$
\left(\left(w, x_{1}\right),\left(w, x_{2}\right),\left(w, y_{1}\right),\left(w, y_{2}\right)\right) \in(W \times(P \sqcup Q))^{(4)}
$$

maps into a certain submanifold of $J_{0}^{(4)}(W \times(P \sqcup Q), N)$ whose codimension is $3 k+2 n$. (That is $3 k$ to make four points of $W$ equal to each other and $2 n$ for two coincidences in $N$.) This codimension is greater than the dimension $4 k+2 p+2 q$ of (the relevant open and closed part of) $(W \times(P \sqcup Q))^{(4)}$, so that transverse means disjoint.

(2) There might exist distinct $x_{1}$ and $x_{2}$ in $P$ and $y$ in $Q$ such that $\Phi\left(w, x_{1}\right)=$ $\Phi(w, y)=\Phi\left(w, x_{2}\right)$. This leads to a submanifold of $J_{0}^{(3)}(W \times(P \sqcup Q), N)$ 
whose codimension is $2 k+2 n$, greater than the dimension $3 k+2 p+q$ of (part of) $(W \times(P \sqcup Q))^{(3)}$.

(3) There might exist $x$ in $P$ and distinct $y_{1}$ and $y_{2}$ in $Q$ such that $\Phi(w, x)=$ $\Phi\left(w, y_{1}\right)=\Phi\left(w, y_{2}\right)$. The relevant manifold has codimension $2 k+2 n$ in $J_{0}^{(3)}(W \times(P \sqcup Q), N)$, greater than $3 k+p+2 q$.

(4) There might exist $x \in P$ and $y \in Q$ such that $\Phi(w, x)=z=\Phi(w, y)$ and such that the linear map $T_{x} P \oplus T_{y} Q \rightarrow T_{z} N$ given by differentiation of $\phi(w)$ at $x$ and $y$ has rank less than $p+q$. For each fixed rank $r<p+q$ this leads to a submanifold of $J_{1}^{(2)}(W \times(P \sqcup Q), N)$ whose codimension $k+(n-r)(p+q-r)$ is greater than $2 k+p+q$.

This completes the proof that the pair $(\mathcal{M}, \mathcal{M}-\mathcal{V})$ is $(2 n-2 p-2 q-1)-$ connected.

To prove that the pair $(\tilde{\mathcal{B}}, \widetilde{\mathcal{B}}-\tilde{\mathcal{V}})$ is $(n-p-q-1)-$ connected, essentially the same kind of standard dimension-counting will succeed, but a simple reference as before to the multijet transversality theorem will not suffice because $\tilde{\mathcal{B}}$ is not simply the space of maps from one manifold to another.

First observe that both the projection $\widetilde{\mathcal{B}} \rightarrow P \times Q \times N$ and its restriction $\widetilde{\mathcal{B}}-\tilde{\mathcal{V}} \rightarrow$ $P \times Q \times N$ are fibrations. It therefore suffices if, for a point $\left(x_{0}, y_{0}, z_{0}\right) \in P \times Q \times N$, the pair $\left(\widetilde{\mathcal{B}}_{0}, \widetilde{\mathcal{B}}_{0}-\widetilde{\mathcal{V}}_{0}\right)$ of fibers is $(n-p-q-1)$-connected. Here $\widetilde{\mathcal{B}}_{0} \subset \mathcal{M}$ is the set of all $\phi$ such that $\phi\left(x_{0}\right)=z_{0}=\phi\left(y_{0}\right)$, and $\widetilde{\mathcal{V}}_{0} \subset \widetilde{\mathcal{B}}_{0}$ is the set of all $\phi$ such that in addition at least one of the following is true:

(1) $\phi(x)=\phi(y)$ for some $x \in P-x_{0}$ and some $y \in Q-y_{0}$.

(2) $\phi(x)=z_{0}$ for some $x \in P-x_{0}$.

(3) $\phi(y)=z_{0}$ for some $y \in Q-y_{0}$.

(4) The linear map $T_{x_{0}} P \oplus T_{y_{0}} Q \rightarrow T_{z_{0}} N$ has rank less than $p+q$.

To deal first with (4), note that $\widetilde{\mathcal{B}}_{0}$ is fibered over the space $\mathcal{L}$ of all linear maps $T_{x_{0}} P \oplus T_{y_{0}} Q \rightarrow T_{z_{0}} N$. Let $\mathcal{L}^{\text {max }} \subset \mathcal{L}$ be the open set of maps of rank $p+q$ and let $\widetilde{\mathcal{B}}_{0}^{\max } \subset \widetilde{\mathcal{B}}_{0}$ be its preimage. The pair $\left(\widetilde{\mathcal{B}}_{0}, \widetilde{\mathcal{B}}_{0}^{\text {max }}\right)$ is $(n-p-q)$-connected (one better than needed), because the pair $\left(\mathcal{L}, \mathcal{L}^{\max }\right)$ is $(n-p-q)$-connected, because the closed set $\mathcal{L}-\mathcal{L}^{\text {max }}$ is the union of finitely many submanifolds having codimension at least $n-p-q+1$.

It remains to show that the pair $\left(\widetilde{\mathcal{B}}_{0}^{\max }, \widetilde{\mathcal{B}}_{0}-\widetilde{\mathcal{V}}_{0}\right)$ is $(n-p-q-1)-$ connected. Both $\widetilde{\mathcal{B}}_{0}^{\max }$ and $\widetilde{\mathcal{B}}_{0}-\widetilde{\mathcal{V}}_{0}$ fiber over $L^{\text {max }}$, so we can replace the two spaces by their fibers, say $\widetilde{\mathcal{B}}_{L}$ and $\widetilde{\mathcal{B}}_{L}-\tilde{\mathcal{V}}_{L}$, over a given $L \in \mathcal{L}$. 
Now given a map $\phi: W \rightarrow \widetilde{\mathcal{B}}_{L}$, we want to perturb it slightly so as to eliminate behaviors (1), (2) and (3). None of these can occur for $x$ near $x_{0}$ or $y$ near $y_{0}$ anyway, given the choice of $L$, so we look for perturbations that are fixed near $x_{0}$ and $y_{0}$. In other words, we look for a small compactly supported change in the map $\Phi: W \times\left(\left(P-x_{0}\right) \sqcup\left(Q-y_{0}\right)\right) \rightarrow N$. This goes as before: case (1) leads to a submanifold of $J_{0}^{(2)}\left(W \times\left(\left(P-x_{0}\right) \sqcup\left(Q-y_{0}\right)\right), N\right)$ with codimension $k+n$, greater than $2 k+p+q$; case (2) leads to a submanifold of $J_{0}^{(1)}\left(W \times\left(\left(P-x_{0}\right) \sqcup\left(Q-y_{0}\right)\right), N\right)$ with codimension $n$, greater than $k+p$; and case (3) leads to a submanifold of $J_{0}^{(1)}\left(W \times\left(\left(P-x_{0}\right) \sqcup\left(Q-y_{0}\right)\right), N\right)$ with codimension $n$, greater than $k+q$.

\section{Normal bundles and homotopy cofibers}

Suppose that $X$ is a smooth manifold, and that the closed subset $Y \subset X$ is a smooth submanifold with normal bundle $v$.

Of course, the Thom space $Y^{v}$ is equivalent to the homotopy cofiber of the inclusion map $X-Y \rightarrow X$. This follows from the fact that there is a homotopy pushout square

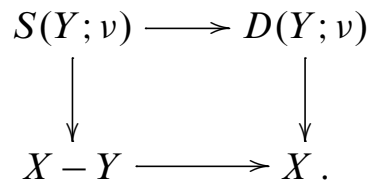

The homotopy fibers over $X$ of the four spaces above form another homotopy pushout square

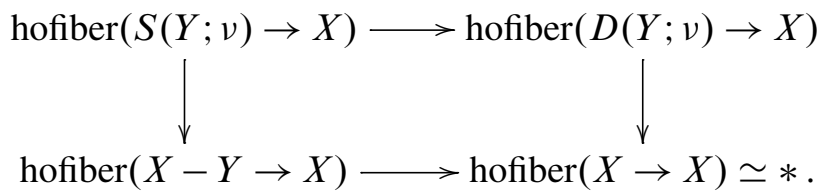

Comparing homotopy cofibers of the rows in this square, we obtain an equivalence

$$
\operatorname{hofiber}(Y \rightarrow X)^{v} \rightarrow \Sigma \text { hofiber }(X-Y \rightarrow X) .
$$

Here we have written $v$ for the pullback of $v$ to hofiber $(Y \rightarrow X)$.

We need statements like those above in which the manifolds $X$ and $Y$ are replaced by the function spaces $\mathcal{M}-\mathcal{V}$ and $\mathcal{B}-\mathcal{V}$ and the role of the normal bundle is played by the vector bundle $T N /(T P \oplus T Q)$ on $\mathcal{B}-\mathcal{V}$. The only little difficulty is that the square (2) depended on having a tubular neighborhood. We will write down a substitute for (2) that avoids this dependence. 
Let $P(Y, X)$ be the space of all smooth paths $\gamma:[0,1] \rightarrow X$ such that $\gamma^{-1}(Y)=0$ and $\gamma^{\prime}(0)$ is not tangent to $Y$. We have the homotopy-commutative square

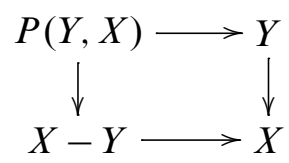

in which the top and left maps are evaluation at 0 and at 1 respectively. There are equivalences

$$
\begin{aligned}
& \operatorname{hocofiber}(P(Y, X) \rightarrow Y) \rightarrow \operatorname{hocofiber}(S(Y ; v) \rightarrow Y)=Y^{v}, \\
& \operatorname{hocofiber}(P(Y, X) \rightarrow Y) \rightarrow \operatorname{hocofiber}(X-Y \rightarrow X)
\end{aligned}
$$

The logic is as follows:

For (4) we use the map $P(Y, X) \rightarrow S(Y ; v)$ that sends $\gamma$ to the projection of $\gamma^{\prime}(0)$ in the direction perpendicular to $Y$, normalized to have unit length. It is a map over $Y$ between two spaces fibered over $Y$, and it is an equivalence because for each point in $Y$ the map of fibers is an equivalence.

For (5) we need to see that the homotopy-commutative square (3) is a homotopy pushout, in the sense that the associated map from the homotopy colimit of

$$
X-Y \leftarrow P(Y, X) \rightarrow Y
$$

to $X$ is an equivalence. After choosing a tubular neighborhood of $Y$ in $X$, one can map $S(Y ; v)$ to $P(Y, X)$ by using radial paths perpendicular to $Y$. This map is an equivalence because it is a one-sided inverse to an equivalence. It follows that in showing that the square is a homotopy pushout we may consider instead the square

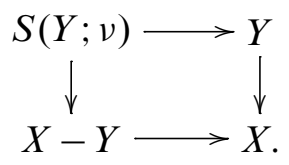

But this comes down to considering the same strictly commutative square (2) that we began with.

Note that although a tubular neighborhood was used in proving (5) to be an equivalence, the definitions of (4) and (5) did not use it. This is the point of introducing $P(Y, X)$.

Now for the function spaces: Again we will obtain equivalences

$$
\operatorname{hocofiber}(P(\mathcal{B}-\mathcal{V}, \mathcal{M}-\mathcal{V}) \rightarrow \mathcal{M}-\mathcal{V}) \rightarrow(\mathcal{B}-\mathcal{V})^{\mathcal{v}}
$$

(where $v$ now means the bundle $T N /(T P \oplus T Q)$ on $\mathcal{B}-\mathcal{V}$ ) and

$$
\operatorname{hocofiber}(P(\mathcal{B}-\mathcal{V}, \mathcal{M}-\mathcal{V}) \rightarrow \mathcal{M}-\mathcal{V}) \rightarrow \operatorname{hocofiber}(\mathcal{M}-\mathcal{B} \rightarrow \mathcal{M}-\mathcal{V})
$$


We define the space $P(\mathcal{B}-\mathcal{V}, \mathcal{M}-\mathcal{V})$. A point in it is a map $\gamma:[0,1] \rightarrow \mathcal{M}$ meeting the following conditions. Write $\gamma(t)=\left(f_{t}, g_{t}\right)$. The conditions are:

(1) $\gamma$ is smooth in the sense that the adjoint maps $(t, x) \mapsto f_{t}(x)$ and $(t, x) \mapsto g_{t}(x)$ from $[0,1] \times P$ and $[0,1] \times Q$ to $N$ are smooth.

(2) For every $t>0, \gamma_{t}$ is in $\mathcal{M}-\mathcal{B}$, that is, $f_{t}(P) \cap g_{t}(Q)=\varnothing$.

(3) $\gamma_{0} \in \mathcal{B}-\mathcal{V}$, that is, (a) there is exactly one point $\left(x_{0}, z_{0}, y_{0}\right) \in P \times N \times Q$ such that $f_{0}(x)=z_{0}=g_{0}(y)$ and (b) $d f_{0} \oplus d g_{0}: T_{x_{0}} P \oplus T_{y_{0}} Q \rightarrow T_{z_{0}} N$ is injective.

(4) $\gamma^{\prime}(0)$ is not tangent to $\mathcal{B}-\mathcal{V}$, that is, the vector $f_{0}^{\prime}\left(x_{0}\right)-g_{0}^{\prime}\left(y_{0}\right) \in T_{z_{0}}(N)$ does not belong to the subspace $\left(D_{x_{0}} f_{0}\right)\left(T_{x_{0}} P\right) \oplus\left(D_{y_{0}} g_{0}\right)\left(T_{y_{0}} Q\right)$. Here $f^{\prime}$ and $g^{\prime}$ are derivatives with respect to $t$.

Consider the homotopy-commutative square

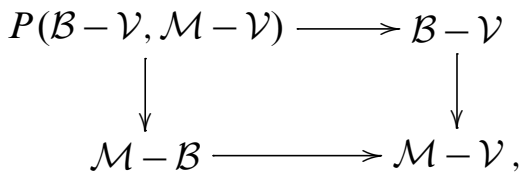

where the upper map and the left map take $\gamma=(f, g)$ to $\left(f_{0}, g_{0}\right)$ and $\left(f_{1}, g_{1}\right)$ respectively. We argue much as in the finite-dimensional case.

First, there is an equivalence $P(\mathcal{B}-\mathcal{V}, \mathcal{M}-\mathcal{V}) \rightarrow S(\mathcal{B}-\mathcal{V} ; v)$ that respects the projection to $\mathcal{B}-\mathcal{V}$, namely the map that takes $\gamma=(f, g)$ to the unit vector in $T_{z_{0}} N /\left(T_{x_{0}} P \oplus T_{y_{0}} Q\right)$ determined by the element $f_{0}^{\prime}\left(x_{0}\right)-g_{0}^{\prime}\left(y_{0}\right)$ of $T_{x_{0}} P \oplus T_{y_{0}} Q$. It is an equivalence because it is a map between spaces fibered over $\mathcal{B}-\mathcal{V}$ and it induces equivalences fiber by fiber.

Second, the square is a homotopy pushout. For this step, instead of trying to come up with a tubular neighborhood we reduce to the finite-dimensional case.

To show that the map from the homotopy colimit of

$$
\mathcal{M}-\mathcal{B} \leftarrow P(\mathcal{B}-\mathcal{V}, \mathcal{M}-\mathcal{V}) \rightarrow \mathcal{B}-\mathcal{V}
$$

to $\mathcal{M}-\mathcal{V}$ is surjective on homotopy groups, let $X=S^{k}$ and take any map $\phi: X \rightarrow$ $\mathcal{M}-\mathcal{V}$, with adjoint $\Phi=(F, G), F: X \times P \rightarrow N, G: X \times Q \rightarrow N$. Deforming by a homotopy that stays within $\mathcal{M}-\mathcal{V}$, make $\Phi$ "transverse to $\mathcal{B}-\mathcal{V}$ " in the sense that $F$ and $G$ together give a map $X \times P \times Q \rightarrow N \times N$ which is transverse to the diagonal. The preimage of the diagonal in $X \times P \times Q$ is a submanifold, and it is embedded in $X$ by the projection. Call its image $Y$. The normal bundle of $Y$ in $X$ is the pullback of $T N /(T P \oplus T Q)$ by $\phi$. 
Now inverting the equivalence

$$
\operatorname{hocolim}(X-Y \leftarrow P(Y, X) \rightarrow Y) \rightarrow X
$$

and composing with the obvious map

$\operatorname{hocolim}(X-Y \leftarrow P(Y, X) \rightarrow Y) \rightarrow \operatorname{hocolim}(\mathcal{M}-\mathcal{B} \leftarrow P(\mathcal{B}-\mathcal{V}, \mathcal{M}-\mathcal{V}) \rightarrow \mathcal{M}-\mathcal{V})$

we get

$$
X \rightarrow \operatorname{hocolim}(\mathcal{M}-\mathcal{B} \leftarrow P(\mathcal{B}-\mathcal{V}, \mathcal{M}-\mathcal{V}) \rightarrow \mathcal{M}-\mathcal{V}),
$$

a lifting (up to homotopy) of $\phi$. Essentially the same argument serves to lift a homotopy and prove the injectivity.

Taking homotopy fibers over $\mathcal{M}-\mathcal{V}$ all around, we obtain the needed equivalence $F$.

Acknowledgments The authors would like to thank Harvard University for their hospitality.

\section{References}

[1] M Golubitsky, V Guillemin, Stable mappings and their singularities, Graduate Texts in Math. 14, Springer, New York (1973) MR0341518

[2] T G Goodwillie, M Weiss, Embeddings from the point of view of immersion theory. II, Geom. Topol. 3 (1999) 103-118 MR1694808

[3] J R Klein, E B Williams, Homotopical intersection theory. I, Geom. Topol. 11 (2007) 939-977 MR2326939

[4] J N Mather, Stability of $C^{\infty}$ mappings. V. Transversality, Advances in Math. 4 (1970) 301-336 (1970) MR0275461

[5] B A Munson, A manifold calculus approach to link maps and the linking number, Algebr. Geom. Topol. 8 (2008) 2323-2353 MR2465743

[6] M Weiss, Embeddings from the point of view of immersion theory. I, Geom. Topol. 3 (1999) 67-101 MR1694812

Department of Mathematics, Brown University

Box 1917, Providence RI 02912-0001

Department of Mathematics, Wellesley College

106 Central Street, Wellesley MA 02481

tomg@math.brown.edu, bmunson@wellesley.edu

http://palmer.wellesley.edu/ munson

Received: 26 October 2009 Revised: 20 February 2010 